\title{
Pedestrian Detectability Estimation Considering Visual Adaptation to Drastic Illumination Change
}

\author{
Yuki IMAEDA ${ }^{\dagger *}$, Nonmember, Takatsugu HIRAYAMA ${ }^{\dagger \dagger a)}$, Yasutomo KAWANISHI ${ }^{\dagger \dagger \dagger b)}$, \\ Daisuke DEGUCHI ${ }^{\dagger \dagger \dagger c)}$, Members, Ichiro IDE ${ }^{\dagger \dagger \dagger)}$, Senior Member, and Hiroshi MURASE ${ }^{\dagger \dagger \mathrm{e})}$, Fellow
}

\begin{abstract}
SUMMARY We propose an estimation method of pedestrian detectability considering the driver's visual adaptation to drastic illumination change, which has not been studied in previous works. We assume that driver's visual characteristics change in proportion to the elapsed time after illumination change. In this paper, as a solution, we construct multiple estimators corresponding to different elapsed periods, and estimate the detectability by switching them according to the elapsed period. To evaluate the proposed method, we construct an experimental setup to present a participant with illumination changes and conduct a preliminary simulated experiment to measure and estimate the pedestrian detectability according to the elapsed period. Results show that the proposed method can actually estimate the detectability accurately after a drastic illumination change.

key words: ITS, driving assistance, pedestrian detectability, illumination change, visual adaptation
\end{abstract}

\section{Introduction}

Most traffic accidents are caused by driver's negligence. The factors in the negligence can fall into recognition, decision, operation, and other errors. Among them, the recognition error is the major factor.

Recently, various methods for pedestrian detection have been proposed [1]. Although automobile manufacturers have started to put them into practical use, overintervention by such driving support is regarded problematic, since they can impede safe driving rather than helping it. Advanced Driving Assistance Systems, therefore, should estimate the pedestrian detectability by the driver and make him/her pay attention only to pedestrians with low detectability.

The pedestrian detectability is defined as the probability of perceiving a pedestrian by the driver [2]. Factors that

Manuscript received September 29, 2017.

Manuscript revised January 24, 2018.

Manuscript publicized February 20, 2018.

${ }^{\dagger}$ The author was with the Graduate School of Information Science, Nagoya University, Nagoya-shi, 464-8601 Japan.

${ }^{\dagger}$ The author is with the Institutes of Innovation for Future Society, Nagoya University, Nagoya-shi, 464-8601 Japan.

${ }^{\dagger \dagger}$ The authors are with the Graduate School of Informatics, Nagoya University, Nagoya-shi, 464-8601 Japan.

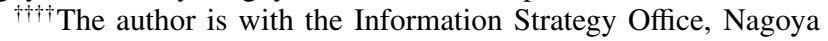
University, Nagoya-shi, 464-8601 Japan.

${ }^{*}$ Presently, with Nissan Motor Co., Ltd.

a) E-mail: takatsugu.hirayama@nagoya-u.jp

b) E-mail: kawanishi@i.nagoya-u.ac.jp

c) E-mail: ddeguchi@ nagoya-u.jp

d) E-mail: ide@i.nagoya-u.ac.jp

e)E-mail: murase@nagoya-u.jp

DOI: 10.1587/transinf.2017EDL8215 change the pedestrian detectability fall into pedestrian's appearance, driver's condition, and traffic environment. The appearance factors include pedestrian's shape, color, motion, etc. Tanishige et al. proposed a pedestrian detectability estimation method focusing on its visual features [3]. The driver's condition factors include fatigue, distraction, aging, etc. Wegner et al. indicated that driver's visual performance declines in intoxicated states [4]. The traffic environment factors include road condition, weather, time of day, etc. Arumi et al. showed that visual acuity worsens according to the road luminance level [5].

In this paper, we focus on drastic bright-to-dark illumination change in the traffic environment as a critical factor of road traffic accidents. Figure 1 shows a simulated example of a driver's vision after the bright-to-dark change. After the change, the driver's visual acuity decreases until adapting to the dark illumination at least several seconds later (dark adaptation) [6]. The findings from such studies in the field of psychophysics have not been applied to pedestrian detectability estimation, since they used simple visual stimuli such as geometric figures for measuring visual sensitivity, while drivers are exposed to more complex visual stimuli in real driving conditions.

The goal of our research is to correctly estimate the detectability of a pedestrian in a traffic scene even after the illumination changes drastically. We focused on the elapsed time from a drastic illumination change as an explanatory factor of the visual adaptation, and in this paper we propose a method to estimate the detectability according to the elapsed time. Our contributions are as follows:

1. Introduction of visual adaptation to the detectability estimation: The proposed method can accurately esti-

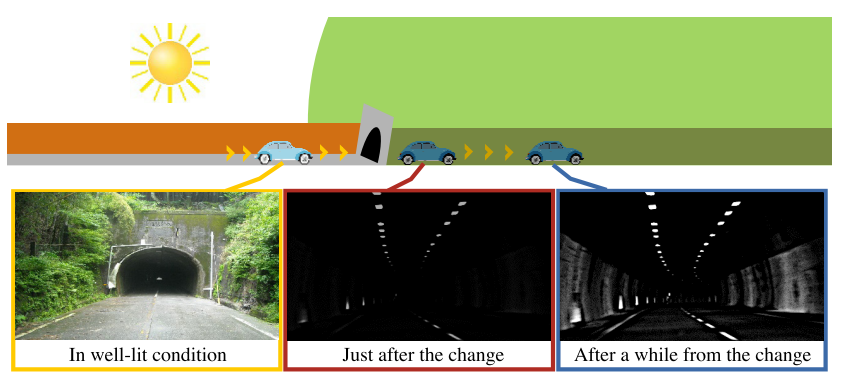

Fig. 1 Simulated example of driver's vision after bright-to-dark illumination change. 
mate the pedestrian detectability by introducing visual feature selection based on the temporal process of the visual adaptation.

2. Dataset construction of the pedestrian detectability with illumination changes: We designed an experimental procedure to obtain the pedestrian detectability with visual adaptation.

\section{Detectability Estimation}

A conventional detectability estimator is based on a simple regression model: $y=\sum_{i} \beta_{i} x_{i}$ where the regression coefficients $\beta_{i}$ are trained from pairs of visual features $x_{i}$ and the ground-truth of the detectability $y$. Since this model cannot handle the temporal process of the visual adaptation, we improve the model as follows:

$$
y(t)=\sum_{i} \beta_{i}(t) x_{i} .
$$

Compared with the conventional estimator, $y$ and $\beta_{i}$ depend on the elapsed time $t$ from illumination change.

In this paper, we construct a discrete model using multiple estimators, which approximates to the above continuous model because the fine-grained dynamics of the groundtruth $y(t)$ can be hardly obtained and the progress of the dark adaptation is relatively slow. Figure 2 shows the process flow of the proposed framework including the multiple estimators. It consists of two phases; training phase and estimation phase.

In the training phase, for each elapsed period $T$, the ground-truth of the detectability $y(T)$ is obtained through a preliminary subjective experiment and an estimator is trained: In Fig. 2, the elapsed period is set to either 0.5, 1.5, or $3.0 \mathrm{sec}$. for example.

In the estimation phase, we first measure the driver's gaze position and extract visual features from an in-vehicle camera image. Next, the drastic illumination change event is detected using a luminometer. We then switch the estimators according to the elapsed period after the event and estimate the detectability at each elapsed period. Here, we employed the visual features used in a conventional method [3] as follows:

\section{(1) Features from the pedestrian region}

In general, the larger and the more complex a pedestrian appears, the easier the driver can detect him/her. Since we assumed that the visual characteristics influence the detectability even after a drastic illumination change, we extracted features related to the size and the appearance of a pedestrian.

$P_{\text {width }}$ and $P_{\text {height }}$ are the width and the height of the rectangle surrounding the pedestrian, respectively. $P_{\sigma(\mathrm{lum})}$ is the standard deviation of luminance in the pedestrian region represented by the rectangle.

(2) Features of the contrast between the appearances of pedestrian region and its surrounding region

The contrast between the appearances of a pedestrian and its surrounding is an important feature considering pedestrian detectability.

$C_{\mu(\text { lum })}$ is the absolute difference between the average luminance in the pedestrian region and that in its surrounding region represented by a rectangle with $P_{\text {width }}+P_{\text {height }}$ and $2 \times P_{\text {height }} . C_{\mu(\mathrm{Lab})}$ is the Euclidean norm between the average color vector in the pedestrian region and that in the surrounding region. $C_{\text {edge }}$ is the absolute difference between the average of edge intensity in the pedestrian region and that in the surrounding region. $C_{\text {hist(color) }}$ is the Earth Mover's Distance between the color histogram in the pedestrian region and that in the surrounding region.

\section{(3) A feature of driver's view angle}

According to psychophysics studies, the visual adaptation in the central fields-of-view differs from that in the periph-

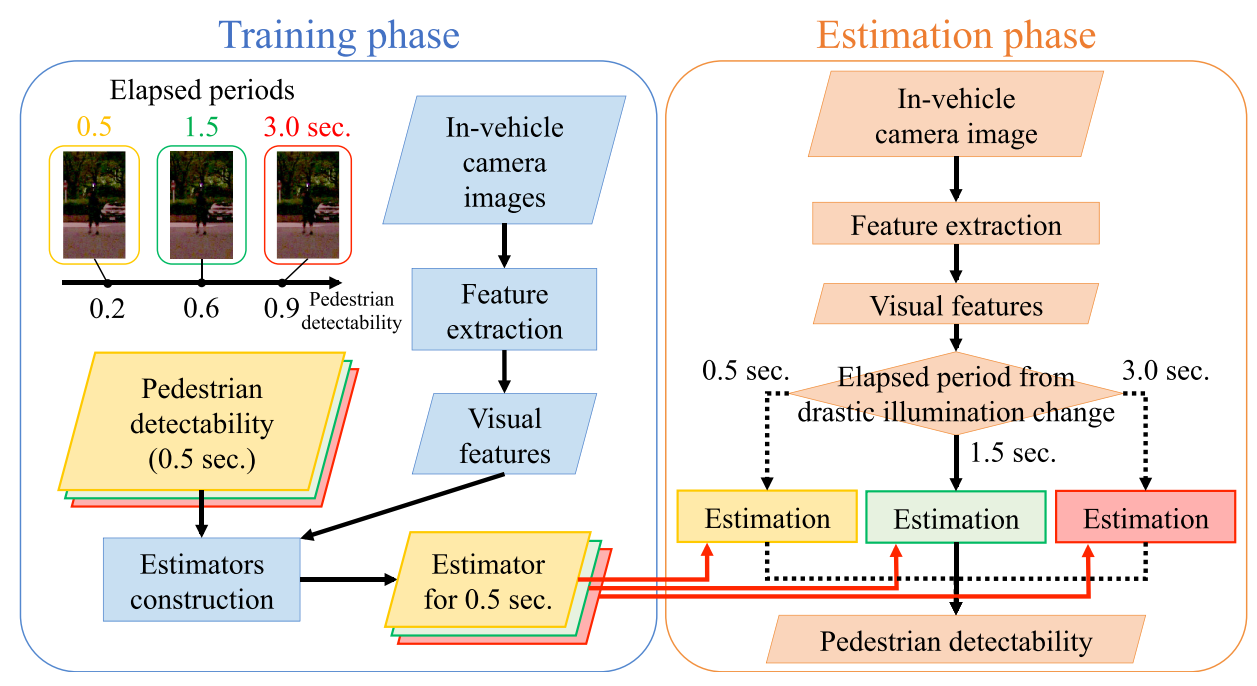

Fig. 2 Process flow of the proposed framework. 
eral fields-of-view. Therefore, we focused on the view angle from the pedestrian to the driver's gaze position. The Euclidean distance between the center of the pedestrian region and the fixation point on an input image is represented as $D_{(p, g)}$.

\section{Dataset Construction}

The pedestrian detectability is the probability that a driver perceives a pedestrian [2]. Since this is the measure based on human sensation, we need to conduct a subjective experiment to obtain the ground-truth before training the estimators. Some works regarded the rate of detecting a pedestrian by experimental participants as the detectability [3]. They were asked to find a pedestrian in an image showing a traffic scene in a stable illumination condition. Since we are focusing on the visual adaptation, we designed an experimental environment to adopt to varying illumination. Figure 3 shows a setup with controllable lights that we implemented to realize the environment. During the experiment, we covered the apparatus and the participant with a blackout curtain to shut out external light. The procedure is described below.

1. The participant adapts to well-lit environment in the setup for $30 \mathrm{sec}$.

2. A low-resolution image showing a traffic scene including a non-detectable pedestrian is displayed to the participant.

3. The participant is instructed to fix his/her eye gaze at a certain position indicated by a cross.

4. The illumination is drastically changed by turning off the lights.

5. After $T$ sec., a high-resolution version of the image is displayed for $0.5 \mathrm{sec}$.

6. The participant searches for a pedestrian.

7. The participant indicates the position of the pedestrian by touching the display.

We presented the low-resolution image of the same scene as the original image in Step 2. to reduce the effect of visual surprise. We conducted the procedure with $T=0.0$, $1.0,2.5,4.0,5.5$, and $7.0 \mathrm{sec}$. For efficiency of time, we displayed two scenes (the first one with, and the second one

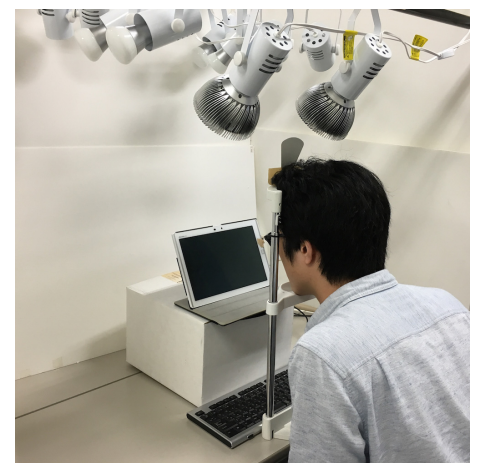

Fig. 3 Experimental setup (Covered with a blackout curtain during the actual subjective experiment). without illumination change) in a single procedure. Here, Steps 2. to 7. except for Step 4. were repeated with the second scene after the cross is displayed again. Figure 4 shows the procedure with three patterns of display timings of two scenes. We obtained the detectability corresponding to six elapsed periods by carrying out the procedure. In addition, we obtained the detectability in full-adaptation to the dark condition (15 min., hereafter $T=\infty$ ).

We used a camera (iVIS HF G10, Canon Inc.) to capture traffic scenes in twilight. Then we manually extracted frames containing one pedestrian from the videos. Figure 5 shows two examples of images whose resolution is $1,920 \times 1,080$ pixels. We also used an organic electroluminescent display (arrows Tab F-03G, Fujitsu Inc., 10.5 inches, 2,560 × 1,600 pixels, 2,000,000:1 contrast ratio) equipped with a touch panel to display the images, and some lights (Grassy LeDiO RX122 FleshWhite, volxjapan Co. Ltd. and Hue, Philips) to control the illumination condition. We fixed the luminance in the well-lit environment to approximately 1,000 luxes, that is, the luminance at an hour before sunset, and that in the dark environment with approximately 10 luxes to reproduce twilight gloom.

We conducted this experiment with eight subjects (four males and four females) with normal vision whose ages ranged between 22 and 30. In this experiment, we prepared 51 images containing just one pedestrian in each of them. Thus, we obtained 357 ground-truth data, i.e., 51 pedestrians for each of seven elapsed periods including $T=\infty$, of the pedestrian detectability. Regarding a criterion to judge whether the participant's response is correct or not, we defined a center region with $P_{\text {width }} / 2$ and $P_{\text {height }} / 2$ in the pedestrian region as ground-truth and a 200 pixels $^{\dagger}$ rectangle around the touched point as the response region. If it overlapped with the ground-truth, it was regarded as correct. Then we calculated the rate of correct responses by all participants as the pedestrian detectability.

Figure 6 shows the relation between the obtained pedestrian detectability and the elapsed period. Here, each bar and line represent the average and the standard error of

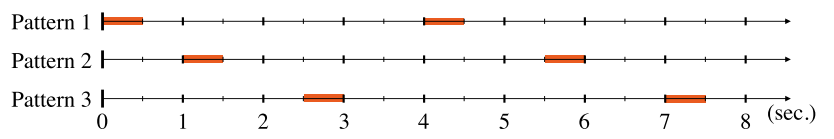

Fig. 4 Patterns of display timings of images after the light is turned off at $0 \mathrm{sec}$. The orange horizontal bar indicates the duration of displaying the image, where we obtain the detectability.
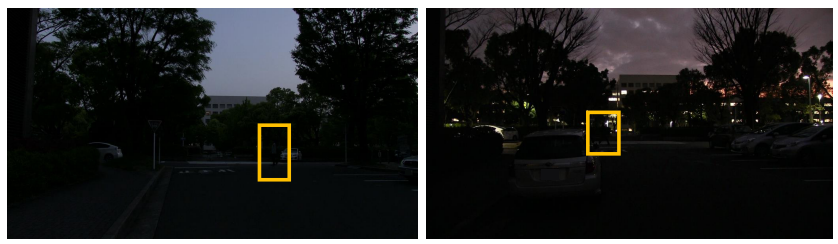

Fig. 5 Examples of images in the dataset.

Corresponds to five degs. in the visual field. 
Table 1 Order of the pair of image features (Evaluated by Mean Squared Error).

\begin{tabular}{|c|c|c|c|c|c|c|c|}
\hline \multirow{2}{*}{ Order } & \multicolumn{7}{|c|}{ Elapsed period [sec.] } \\
\hline & 0.5 & 1.5 & 3.0 & 4.5 & 6.0 & 7.5 & $\infty$ \\
\hline 1 & $\begin{array}{c}P_{\delta(\text { lum })} \cdot C_{\text {edge }} \\
0.028\end{array}$ & $\begin{array}{c}P_{\delta(\text { lum })} \cdot C_{\mu(\text { lum })} \\
0.069\end{array}$ & $\begin{array}{c}P_{\delta \text { (lum) }} \cdot C_{\mu(\text { lum })} \\
0.051\end{array}$ & $\begin{array}{c}P_{\delta(\text { lum })} \cdot C_{\mu(\text { lum })} \\
0.062\end{array}$ & $\begin{array}{c}P_{\delta(\text { lum })} \cdot C_{\mu(\text { lum })} \\
0.056\end{array}$ & $\begin{array}{c}P_{\delta(\text { lum })} \cdot C_{\mu(\text { lum })} \\
0.071\end{array}$ & $\begin{array}{c}P_{\delta(\text { lum })} \cdot C_{\mu(\mathrm{Lab})} \\
0.086\end{array}$ \\
\hline 2 & $\begin{array}{c}P_{\delta(\mathrm{lum})} \cdot C_{\mu(\mathrm{lum})} \\
0.031 \\
\end{array}$ & $\begin{array}{c}P_{\delta(\text { lum })} \cdot C_{\text {edge }} \\
0.081\end{array}$ & 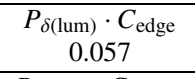 & $\begin{array}{c}P_{\delta(\text { lum })} \cdot C_{\text {edge }} \\
0.063\end{array}$ & $\begin{array}{c}P_{\delta(\text { lum })} \cdot C_{\text {edge }} \\
0.075\end{array}$ & $\begin{array}{c}P_{\delta(\mathrm{lum})} \cdot C_{\mu(\mathrm{Lab})} \\
0.075\end{array}$ & $\begin{array}{c}P_{\delta(\text { lum })} \cdot C_{\mu(\text { lum })} \\
0.094\end{array}$ \\
\hline 3 & $\begin{array}{c}C_{\mu(\mathrm{Lab})} \cdot C_{\text {edge }} \\
0.039\end{array}$ & $\begin{array}{c}C_{\mu(\mathrm{Lab})} \cdot C_{\text {edge }} \\
0.084\end{array}$ & $\begin{array}{c}P_{\delta(\text { lum })} \cdot C_{\mu(\mathrm{Lab})} \\
0.064\end{array}$ & $\begin{array}{c}P_{\delta(\text { lum })} \cdot C_{\mu(\mathrm{Lab})} \\
0.080\end{array}$ & $\begin{array}{c}P_{\delta(\text { lum })} \cdot C_{\mu(\mathrm{Lab})} \\
0.080\end{array}$ & $\begin{array}{c}P_{\text {height }} \cdot C_{\mu(\mathrm{Lab})} \\
0.095\end{array}$ & $\begin{array}{c}P_{\text {width }} \cdot C_{\mu(\text { lum })} \\
0.111\end{array}$ \\
\hline
\end{tabular}

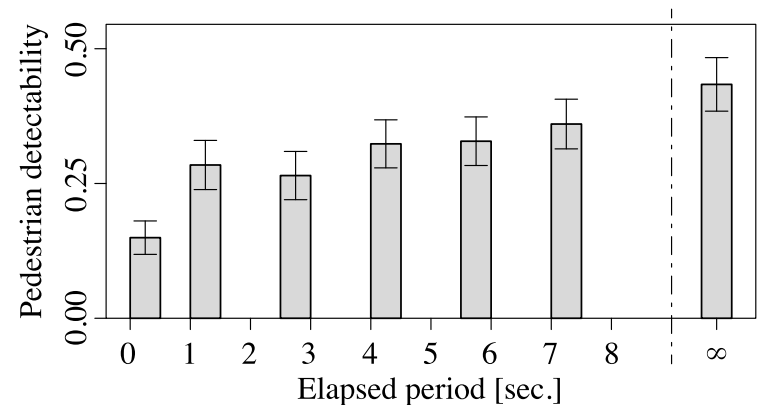

Fig. 6 Pedestrian detectability for each elapsed period. The error bar indicates the standard error calculated from the detectabilities of 51 pedestrians.

the pedestrian detectability of 51 pedestrians, respectively. From this result, we can say that the pedestrian detectability increased gradually according to the dark adaptation in this experiment.

\section{Result and Discussion}

To construct the multiple estimators for the proposed method, we divided the dataset into seven groups according to the elapsed periods including $T=\infty$ and trained an estimator for each group. We employed Support Vector Regression to construct the estimators. To evaluate the proposed method considering the visual adaptation, we compared the estimation error with a comparative method which used a single estimator trained with the detectability data in which the elapsed period $T$ was $\infty$ [3]. The number of data to train the single estimator was the same as that to train each of the multiple estimators. Leave-one-pedestrian-out cross validation was applied; 50 pedestrians were used for training and one pedestrian was used for testing.

Figure 7 shows the comparison of the estimation error for each elapsed period between the proposed method and the comparative method. Note that the estimation errors of two methods in which the elapsed period $T$ was $\infty$ were matched because they used the same training data. Except for $T=\infty$, the proposed method had lower error than the comparative method.

For discussion, we analyzed the effectiveness of the features for each elapsed period. In detail, we first chose a couple of features from the eight visual features, then constructed estimators corresponding to the elapsed period and evaluated them. This procedure was applied to all combinations of features $\left({ }_{8} \mathrm{C}_{2}=28\right)$. Finally we placed them in

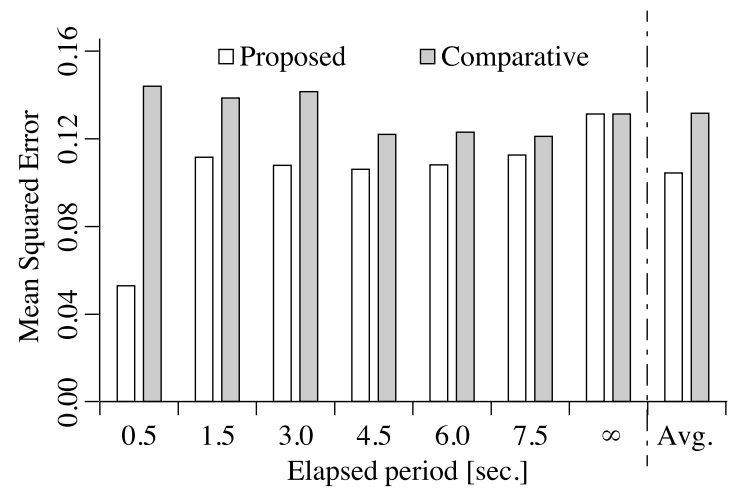

Fig. 7 Estimation errors of the pedestrian detectability.

ascending order of the estimation error as shown in Table 1. This table indicates that the combination $P_{\delta(\text { lum })} \cdot C_{\mu(\text { lum })}$ contributed to achieve lower estimation error over all elapsed periods. Also, we can see that the shorter the elapsed period, the higher the contribution of the combination $P_{\delta(\text { lum })} \cdot C_{\text {edge }}$, and the longer the elapsed period, the higher the contribution of the combination $P_{\delta(\mathrm{lum})} \cdot C_{\mu(\mathrm{Lab})}$. This result indicates the participants' visual system recovered the sensitivity of color as the visual adaptation progressed.

\section{Conclusion}

In this paper, we attempted to estimate the pedestrian detectability considering visual adaptation to drastic illumination change. Specifically, we focused on time elapsed from the illumination change and proposed a method to estimate the detectability according to the elapsed time.

To evaluate the proposed method, we first implemented an experimental setup to present a participant with illumination changes and then conducted an experiment to measure and estimate the detectability according to different elapsed periods. Evaluation results showed that the proposed method considering the visual adaptation was effective, although they are still preliminary results shown through a small and simulated experiment.

As future work, we will introduce additional features based on physiological knowledge, and conduct the subjective experiment to expand the dataset. We have not conducted an experiment in any real traffic environment since it is difficult to control the illumination condition using current technologies in various outdoor scenes. However, we are interested in comparative analysis of results obtained in real and simulated environments in the future. 
Furthermore, we will apply the obtained knowledge to an actual vehicle to validate the application possibility. Specifically, the vehicle will measure the difference in luminance before and after a drastic illumination change, detect a pedestrian by using a camera and a distance measuring sensor, and estimate the pedestrian detectability according to the elapsed time from the drastic illumination change. When the difference is larger than a threshold, the vehicle will promote the driver to recover the visual acuity by turning the low beam headlight on. However, this does not always help the driver to find the pedestrian. If the pedestrian has low detectability, the vehicle projects additional light to only the pedestrian using a controllable headlight device in consideration of the arrival time to it. In the case that the driver could not react to the light projection, the vehicle autonomously avoids the pedestrian. To realize such a vehicle, we need to conduct an experiment with various differences in luminance and construct a computational model of pedestrian detectability considering the difference and the elapsed time.

\section{Acknowledgements}

Parts of this research were supported by JSPS Grantin-Aid for Scientific Research, MEXT under contract no. $17 \mathrm{H} 00745$.

\section{References}

[1] P. Dollár, C. Wojek, B. Schiele, and P. Perona, "Pedestrian detection: An evaluation of the state of the art," IEEE Trans. Pattern Anal. Mach. Intell., vol.34, no.4, pp.743-761, April 2012.

[2] D. Engel and C. Curio, "Detectability prediction in dynamic scenes for enhanced environment perception," Proc. 2012 IEEE Intell. Vehicles Symp., pp.178-183, June 2012.

[3] R. Tanishige, D. Deguchi, K. Doman, Y. Mekada, I. Ide, and H. Murase, "Prediction of driver's pedestrian detectability by image processing adaptive to visual fields of view," Proc. 17th IEEE Int. Conf. Intell. Transport. Syst., pp.1388-1393, Oct. 2014.

[4] A.-J. Wegner and M. Fahle, "Alcohol and visual performance," Prog. Neuro-Psychopharmacol. and Biol. Psychiatry, vol.23, no.3, pp.465-482, April 1999.

[5] P. Arumi, K. Chauhan, and W.N. Charmant, "Accommodation and acuity under night-driving illumination levels," Ophthalmic Physiol. Opt., vol.17, no.4, pp.291-299, July 1997.

[6] W.A.H. Rushton, "Rhodopsin measurement and dark-adaptation in a subject deficient in cone vision," J. Physiol., vol.156, no.1, pp.193-205, April 1961. 